\title{
Dog phobia in a motion-blind patient
}

\author{
O. Blanke, V. Vaclavik, T. Landis, and A.B. Safran \\ University Hospital of Geneva, Switzerland
}

\begin{abstract}
Introduction. A prominent neurophysiological model of phobia generation holds that specific phobia might result from the uncoupling of unaware subcortical fear responses from aware cortical fear responses. Former responses are thought to be automatic and fast, providing approximate information about the external stimulus, whereas the latter responses are more controlled and allow comparison with previous experience. Since only the cortical pathway carries information available to awareness, this model also accounts for the striking irrationality of specific phobia in humans.

Methods. Here, we report neuropsychological and neuro-opthalmological findings in a 41-year-old patient who developed severe dog phobia following bilateral parietal lobe damage.

Results. The examinations showed a severe deficit in visual motion perception (visual motion blindness or akinetopsia) as well as spatial vision. Importantly, the patient was largely unaware of his visual deficits.

Conclusion. Based on the present observation it is argued that irrational fear, as found in specific phobia, might not only result from a general uncoupling of aware cortical from unaware subcortical fear responses, but also from a functionally similar dissociation at the cortical level.
\end{abstract}

A specific phobia is a marked and persistent fear, which is excessive or unreasonable, brought about by the presence or anticipation of a specific object or situation (DSM-IV, diagnostic code 300.29). Although specific phobias have been of lesser public health and clinical interest than other anxiety disorders, their circumscribed nature (e.g., fear of dogs) make them a productive subject for research into fear-inducing brain mechanisms (Fyer, 1998). Based on the pivotal role of the amygdala in fear processing and its abundant afferent input from subcortical and cortical areas concerning external stimuli (Amaral, Price,

Correspondence should be addressed to Dr Olaf Blanke, Department of Neurology, University Hospital of Geneva, 24 rue Micheli-du-Crest, 1211 Geneva, Switzerland; email: olaf.blanke@hcuge.ch

The authors would like to thank the two anonymous reviewers for their helpful suggestions. This research was supported by Swiss National Science Foundation grants 3100-0678774.02 and 3100065096.01 .

(C) 2003 Psychology Press Ltd

http://www.tandf.co.uk/journals/pp/13546805.html

DOI:10.1080/13546800244000300 
Pitkanen, \& Carmichael, 1992), LeDoux (1996) has proposed a neurophysiological model of phobia generation. Phobia is assumed to result from the uncoupling of automatic subcortical fear responses (thalamus-amygdala pathway) from more controlled cortical fear responses (thalamus-cortex-amygdala pathway), which normally operate in a parallel and complementary fashion (Figure 1A). The subcortical pathway is assumed to provide fast and approximate information about the stimulus and thereby establishes emotional memories. The cortical pathway is slower, more exact, and enables comparison with previous experience. Since only the cortical pathway carries information available to awareness, this model also accounts for the striking irrationality of phobia in humans (Fyer, 1998). Yet, much less is known about the contribution of the different cortical areas in fear and phobia generation. Although some evidence suggests that the medial prefrontal cortex might influence fear processing via an inhibition of amygdala responsiveness (Bremner et al., 1999; LeDoux, 1996)), not much is known about other cortical areas. Moreover, there are, to our knowledge, no descriptions of patients with specific phobia subsequent to focal cortical lesions, although pathological fear processing in humans has been described following damage to the amygdala (Adolphs, Tranel, Damasio \& Damasio, 1994; Aggleton, 1992; Calder et al., 1996).

Here, we report on a patient who developed dog phobia following bilateral parieto-occipital brain damage. Dog phobia was accompanied by severe visuospatial and akinetopsic cognitive deficits, which led to the visuospatial misperception of consciously recognised objects. Since the patient was largely unaware of his cognitive deficits, the present observation suggests that irrational fear, as found in specific phobia, might not only result from a general uncoupling of aware cortical from unaware subcortical fear responses (LeDoux, 1996), but also from a functionally similar disassociation at the cortical level.

\section{CASE REPORT}

Patient MB was a 41-year-old, right-handed male admitted to the hospital for fluctuating walking problems and unusual occipital headache. The neurological examination revealed discrete right-sided paresis and hypoesthesia.

\section{Methods and results}

Thorough neuropsychological testing showed optical ataxia, constructional apraxia, and Gerstmann syndrome (digital agnosia, acalculia, agraphia, right-left perturbations). Digital agnosia was found for both hands (the patient was asked to indicate verbally which finger was touched (five trials for each hand): 3 false responses for left-hand and 4 for right-hand testing). Acaculia showed numerous errors for addition, subtraction, and multiplication (division not tested). Agraphia was moderate and found for both hands. Right-left orientation was also perturbed but not investigated psychometrically. Object naming was deficient 


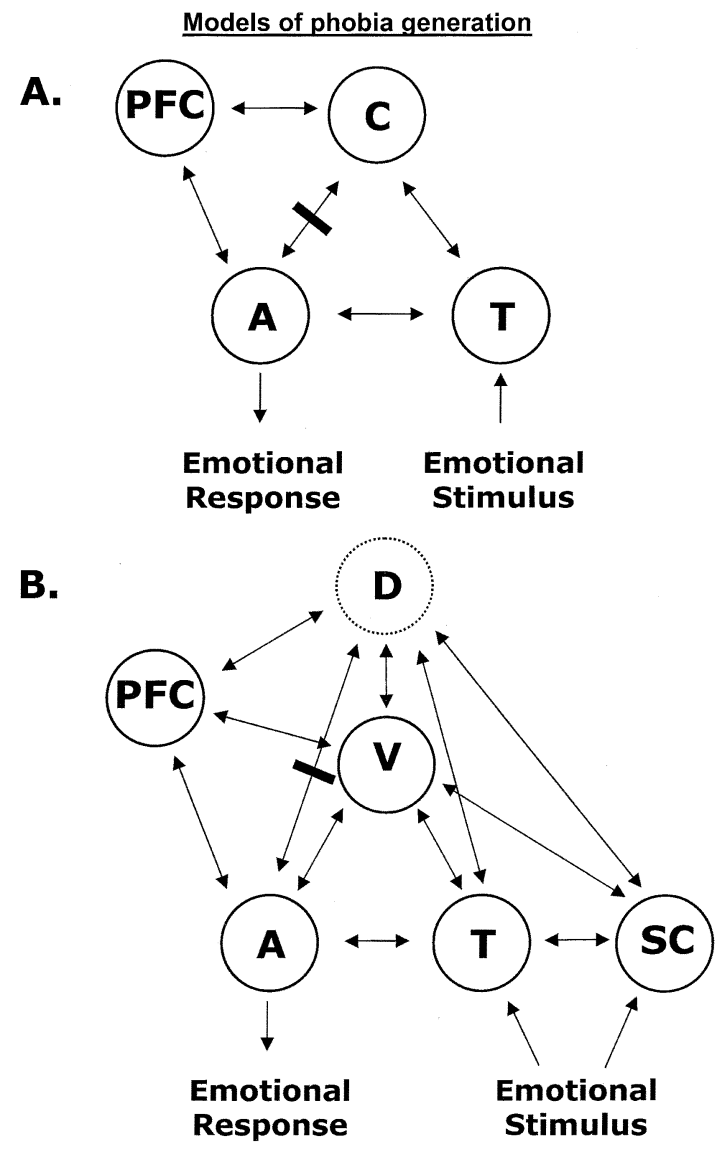

Figure 1. (A) Graphical representation of LeDoux's (1996) model of phobia generation. Phobia is assumed to result from the uncoupling of subcortical fear responses (thalamus-amygdala pathway) from cortical fear responses (thalamus-cortex-amygdala pathway), which normally operate in parallel. The potential role of the ventral prefrontal cortex on phobia is also indicated (see text). Following this model, phobia results from the fact that emotional stimuli reach the amygdala directly from the thalamus but not via the cortex. A, amygdala; T, thalamus; PFC, prefrontal cortex; C, cortex other than PFC. Bidrectional arrows indicate reciprocal connections. The intersected line (black bar) indicates the pathway, whose disconnection is supposed to lead to phobia. (B) Extended model of phobia generation based on the findings in patient $\mathrm{MB}$ and recent neuroimaging evidence on a subcortical fear pathway via the superior colliculus (SC; Morris et al., 1999, 2001; see text). It is suggested that dog phobia in patient $\mathrm{MB}$ resulted from the partial uncoupling of cortical fear responses in the parietal lobe (dorsal stream mediated; D encircled by a dotted line) from intact cortical fear responses in the temporal lobe (ventral stream mediated; V) and intact subcortical fear responses (thalamus and superior colliculus mediated). Phobia is assumed to result from the fact that emotional stimuli reach the amygdala via the thalamus/superior colliculus and the ventral stream but not from the dorsal stream. Other alternatives for phobia generation are discussed in the text. Abbreviations and symbols as in Figure 2A. 
(Boston Naming Test: 24 of 34 items correct). In addition, an impairment for the recognition of fragmented visual stimuli was found (Hooper Visual Organisation Test; 6 of 20 items correct). There were moderate signs of anosognosia without signs of left-sided extinction or neglect (line bisection; no significant deviation of the mean; 20 trials). These deficits contrasted with visual recognition of single objects ( 5 items correct), images ( 5 items correct), famous people ( 6 items correct), as well as superimposed images (Poppelreuter overlapping figure test: all items correct), which were strikingly normal. Moreover, there were no difficulties recognising faces (Benton Face Recognition Test; 25 of 27 items correct) or naming of colours. Digit Span (5 digits) and Corsi Block Span (3 blocks), as well as verbal memory (Rey Auditory Verbal Learning Test; total of first five recalls: 47; delayed recall: 11) were impaired. Autobiographical memory was normal.

Magnetic resonance imaging (MRI) showed a recent left posterior parietal lesion including the superior and inferior parietal lobule as well as the supramarginal gyrus that extended into the adjacent occipital lobe. A smaller and older, previously undetected infarction, was detected in the right superior and inferior parietal lobule (Figure 2).

During the first months after discharge from the hospital, patient MB continued physio- and ergotherapy three times a week and frequently went on long walks. Though not afraid of dogs, other animals, or specific situations prior to hospitalisation, patient $\mathrm{MB}$ started to experience immediate and excessive fear
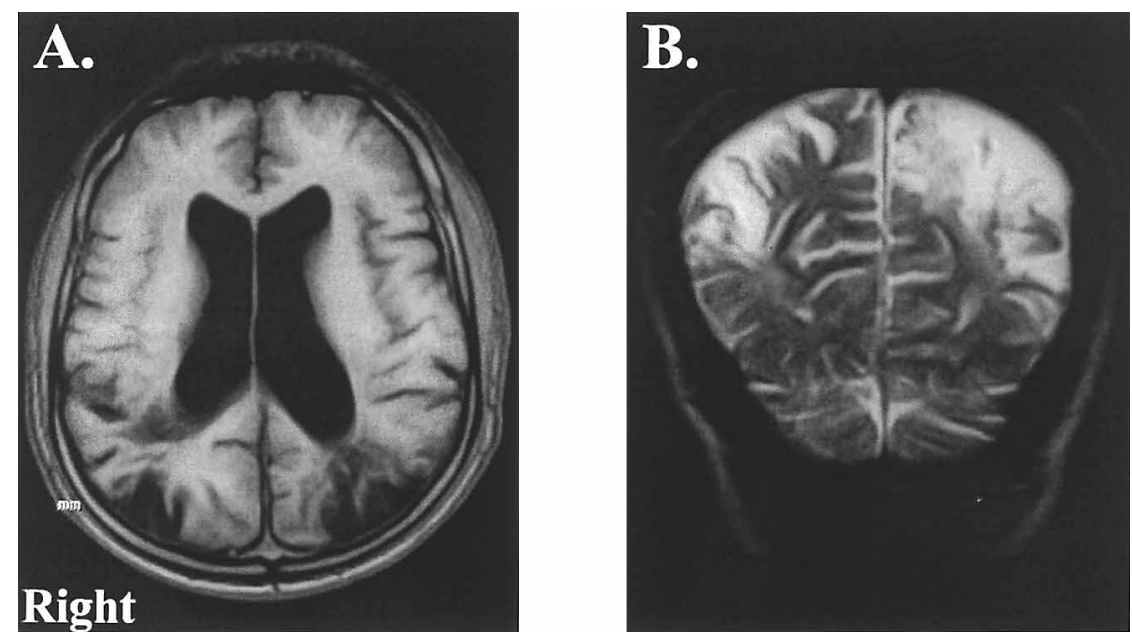

Figure 2. T1-weighted magnetic resonance imaging (MRI) depicting the bilateral lesion in patient MB (transverse plane, Figure 1A). Note predominance in the left hemisphere and the restriction to the parietal lobes with an extension towards the occipital lobe, which can also be appreciated in Figure 1B (coronal plane, T2-weighted MRI). 
when encountering dogs. Fear was felt independently of the size of the dog. It was mostly experienced when the dog approached or passed the patient while he was walking or jogging. Although excessive fear was not experienced for other objects or situations, he also noted that he felt uncomfortable when walking in a crowded street or supermarket. Later interviews revealed that he generally had difficulties judging the speed, direction, and position of moving visual objects as well as their spatial relation to himself (objects included people, cars, and dogs). Difficulties with respect to the judgement of car speed were quite important and led to problems crossing a street safely. However, while his difficulties with visual objects progressively diminished during the first year following hospitalisation, his dog fear worsened to the point that he eventually stopped jogging and finally avoided going out of his house as much as possible. Patient MB realised the irrationality of his dog fear, particularly since he was always very fond of dogs and even owned two dogs as a child. Although he recalls having been bitten once as a child by one of his dogs, neither as a child nor as an adult has he ever been afraid of dogs.

Patient MB did not relate the specific dog fear to his brain damage, but consulted his physician where the neurological control examinations as well as a control MRI were not modified with respect to previous examinations. However, a neuro-ophthalmological examination revealed a severe deficit for the detection and discrimination of visual motion (described in detail below), whereas other parts of this examination were normal or only slightly impaired. Thus, corrected visual acuity was normal for both eyes $(-0.75)$. Ocular tension was normal at $12 \mathrm{mmHg}$ for the right eye and $14 \mathrm{mmHg}$ for the left eye. Pupillary reaction was normal as was corneal sensitivity. There was no diplopia. The initiation of voluntary eye movements was slowed in the vertical and horizontal directions. The fundus of both eyes was normal. Visual fields were measured with an OCTOPUS 2000R automated perimeter (Interzeag AG, Switzerland) and showed mild bilateral peripheral visual field defects (Figure 3A).

Visual motion discrimination. This was tested by the use of a random dot display and a five-alternative forced-choice discrimination task as described previously (Blanke, Landis, Safran, Seeck, 2002; Losey, Safran, Mermoud, Michel, \& Landis, 1998). Coherent motion stimuli (random dot cinematograms) were presented on a 20 inch computer monitor (Sony; frame rate, $70 \mathrm{~Hz}$; $640 \times 480$ pixels) in black and white in a normally lit room. Viewing distance was $50 \mathrm{~cm}$. The stimuli were presented in a borderless square of $12^{\circ} \times 12^{\circ}$ in the central visual field. Each random dot field contained 1000 dots (diameter: $0.68^{\circ}$ ). The dots had a random duration between $4 \mathrm{~ms}$ and $800 \mathrm{~ms}$ and were randomly positioned within the square. A percentage of the dots was programmed to be displaced with a velocity of $2 \%$ second in the tested direction (signal dots). The percentage of coherence motion $(\% \mathrm{CM})$ was defined as the number of signal dots divided by the total number of dots and multiplied by 100 


\section{Visual Perimetry}
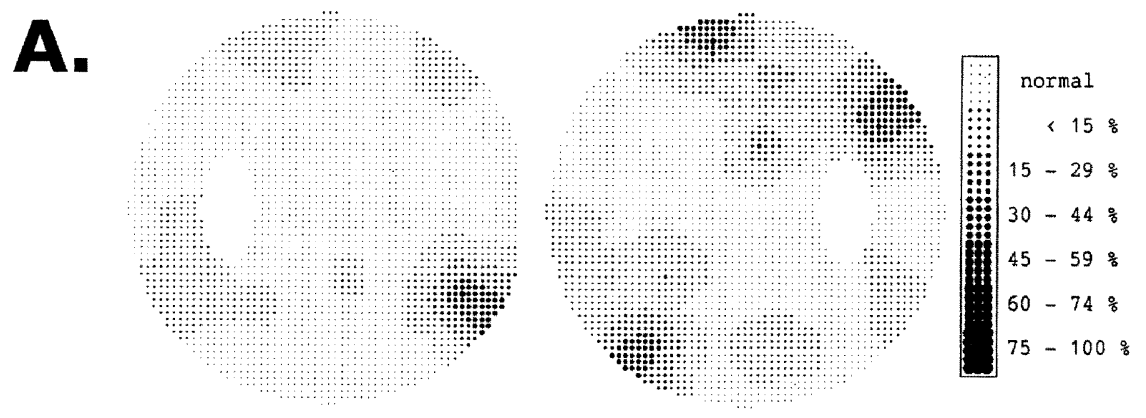

B.

\section{Visual motion stimuli}

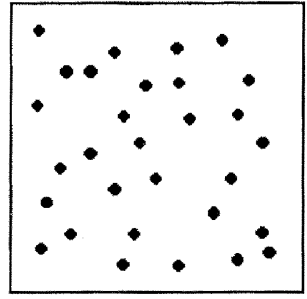

$0 \% \mathrm{CM}$

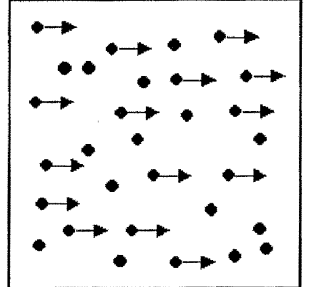

$50 \% \mathrm{CM}$

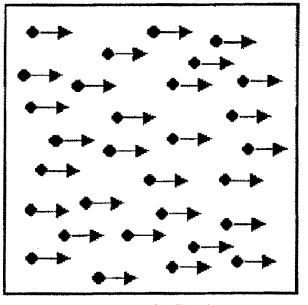

$100 \% \mathrm{CM}$

Figure 3. (A) Visual fields for right eye (on the right) and left eye (on the left) as measured by OCTOPUS 2000R static perimeter. Only mild (highest elevation of $30-40 \%$ of maximal intensity) and peripheral visual field defects were found. Size of the dots depicts the percentage of the maximal stimulus intensity (1000 apostilb) that was necessary for the test $\operatorname{spot}\left(0.41^{\circ}\right.$ in diameter) to be perceived at the indicated location. The central $30^{\circ}$ of visual angle are shown for both eyes. (B) The visual motion stimuli for motion in the frontal plane are illustrated. Black dots with arrow indicate signal dots, dots without arrows noise dots. The arrows indicate the direction of dot movement. At $100 \%$ coherent motion (CM) all dots move in the same direction (right). With decreasing \% $\mathrm{CM}$ levels the percentage of dots moving in the tested direction decreases, i.e., $50 \% \mathrm{CM}$ (middle) or $0 \% \mathrm{CM}$ (left).

(Figure 3B). Dots moving out of the stimulus area reappeared on the opposite side. The direction of each RDC stimuli in each block was varied randomly between the four cardinal directions (right-left-up-down). An automated staircase algorithm varied the $\% \mathrm{CM}$, starting at $100 \% \mathrm{CM}$ (all dots moving in one direction). The patient replied whether he perceived motion as right, left, up, down, or that he did not perceive the direction of motion (i.e., five-alternative forced-choice paradigm). If the answer was correct, the \%CM was decreased, if 
not, \% $\mathrm{CM}$ was increased. The staircase was continued until five response reversals had occurred. The mean of the last three reversals was taken as the \%CM threshold (Blanke et al., 2002; Losey et al., 1998). Psychophysical thresholds for motion in depth were tested in the same way. The stimuli were presented in a larger square of $22^{\circ} \times 16^{\circ}$ in the central visual field. The direction of each random dot cinematogram (RDC) stimulus was varied randomly between apparent motion towards or away from the patient (three-alternative forced-choice paradigm: towards, away, no motion perceived). Other stimulus characteristics were as described above for motion in the frontal plane. In both tasks, the patient was instructed to look at the centre of the screen and to retain from eye movements. Fixation was monitored by the examiner and trials rejected if fixation was not maintained. No feedback about correctness of the response was provided. The patient gave his answer aloud and the examiner recorded the response. The rate of trial presentation was controlled by the examiner and adjusted to patient comfort. The psychophysical threshold was determined as the mean of the thresholds for each of the tested directions. The psychophysical thresholds for motion in the frontal plane in healthy control subjects was found at $0.8 \pm 0.2 \% \mathrm{CM}$ (mean \pm standard deviation) and for motion in depth at $0.3 \pm 0.1 \% \mathrm{CM}$ (see Losey et al., 1998). A \%CM threshold falling above this limit was considered a pathological threshold elevation. The psychophysical thresholds for the discrimination of visual motion in the frontal plane in patient $\mathrm{MB}$ was severely increased to $52.0 \% \mathrm{CM}$ as was the threshold for visual motion in depth $(48.7 \% \mathrm{CM})$. Visual motion perception was controlled a month later and confirmed the severity of the visual motion deficit (psychophysical threshold for motion in the frontal plane was at 57.0\% CM). Based on these neuropsychological and neuro-ophthalmological results and the interview, it was explained to the patient that the brain damage impaired his ability to judge the direction and position of visual objects including dogs. This fact relieved the patient and diminished progressively his dog fear in subsequent dog encounters. Nevertheless, patient MB has still remained afraid of dogs and avoids approaching them as much as possible.

\section{DISCUSSION}

Several arguments suggest that dog phobia in patient MB resulted from posterior parietal brain damage. First, patient MB developed dog phobia within months following the onset of the second stroke and was never afraid of dogs previously (although he remembers having been bitten by a dog during childhood). Second, the specific cognitive deficits in patient MB might provide an explanation for his dog fear. The processing of spatial attributes of visual objects was severely impaired, while he could correctly recognise and name the same objects. The impaired functions are important for the perception of speed, direction, and position of external objects with respect to oneself as well as to programme 
appropriate actions (Grüsser \& Landis, 1991; Milner \& Goodale, 1995). Thus, patient MB could recognise a dog, but would constantly misperceive its position and direction of motion and would probably misplan appropriate behaviour. Third, previously described patients suffering from the rare condition of motion blindness, or akinetopsia, also experienced fearful situations related to the misperception of visuospatial stimulus attributes (Goldstein \& Gelb, 1918; Zihl, von Cramon, \& Mai, 1983). Importantly, as in patient MB, both previously reported patients described their misperceptions only when recognising their motion perception deficit during extensive testing, and had not considered them to be related to their brain disorder. These two patients reported difficulties in perceiving the position and direction of people walking in a room. This problem was enhanced when walking in the street, which was consequently avoided as much as possible (Zihl et al., 1983). The patient of Goldstein and Gelb (1918) reported difficulties recognising the position of an approaching streetcar, which he did not perceive as moving, and had difficulties judging the motion and distance of other pedestrians with respect to his position. Fourth, patient MB's lesion location is consistent with localisation of visuospatial and visuomotor function in normals and previous motion-blind patients. Patient MB's brain damage was largely restricted to the parietal areas and thus to the dorsal stream of visual processing ("where"-pathway; see Ungerleider \& Mishkin, 1982), while brain areas involved in the processing of identity attributes of visual objects (e.g. recognition) in the temporal lobe (ventral stream) remained intact ("what"-pathway; Ungerleider \& Mishkin, 1982). Moreover, brain damage was bilateral as in previous motion-blind patients (Goldstein \& Gelb, 1918; Zihl et al., 1983). Fifth, patient MB was only partially aware of his cognitive deficits, similar to both previously reported motion-blind patients. This might explain the poor insight and the persistence of his dog phobia, which is characteristic in patients with specific phobias (Fyer, 1998).

Patient MB also felt uncomfortable when walking in crowded streets (people) or when crossing a street (cars) without the presence of dogs and avoided these situations as much as possible, but did not become phobic for people or cars.

Although we do not know exactly why patient MB suffered from phobia specifically for dogs, it might be suggested that the early aversive encounter during childhood (the patient was bitten by a dog) plays a potential role (Fyer, 1998). LeDoux (1996) has suggested that the acquired fear responses are indelible, but may be extinguished by subsequent nonfearful encounters of the initial phobic situation (encounters with dogs without being bitten). The present patient's cognitive impairments due to brain damage might have thus reinstated the fear response that was acquired much earlier.

Alternatively, fear of dogs (or other dog-sized animals) might represent an evolutionary determined biological preparedness for fear generation (avoidance of situations with dog-sized animals) (Fyer, 1998; Seligman, 1971), which is under cortical control (LeDoux, 1996). This is also suggested by the prevalence 
of dog phobia and the fact that only a small number of stimuli accounts for most human phobias (Agras, Chapin, \& Oliveau, 1972). Both arguments suggest that dog phobia in the present patient is more likely to occur than phobia for other kinetic objects, such as cars, while crossing a street. We can only speculate how patient MB's cognitive impairments led to specific object-related phobia. First it is suggested that pathological and unaware spatial processing in the dorsal stream, in combination with normal and aware object-related processing mediated by the ventral stream, may have played the causal role in the generation of specific dog phobia in patient MB. In other words, misperceived visuospatial information about a consciously recognised object (dog) is continuously relayed to the amygdala. This chronically ambiguous input from both cortical streams might modify over time the controlling role that both cortical pathways normally (previous to brain damage) exert upon sensory emotional information that arrives directly in the amygdala (LeDoux, 1996; see Figure 1B). Second, amygdala processing might have also been disrupted by ambiguous input from both cortical streams to the medial prefrontal cortex. The latter structure is important in processes of fear extinction (Bremner et al., 1999; LeDoux, 1996) and a disruption of the prefrontal mechanisms of inhibition and modulation upon amygdala processing might also have lead to dog phobia. The present observation thus suggests that chronic and ambiguous input from both cortical streams might directly or indirectly result in pathological (and probably exaggerated) amygdala activation as observed in patients with anxiety/phobic disorders in response to unmasked fearful stimuli (Liberzon et al., 1999; Shin et al., 1997), masked fearful stimuli (Rauch et al., 2000), and neutral stimuli (Birbaumer et al., 1998). Amygdala activation for visual fearful stimuli was also revealed in a patient with affective blindsight following extensive occipital damage (Morris, DeGelder, Weiskrantz, \& Dolan, 2001). This patient was able to discriminate emotional faces presented in his blind hemifield (contralateral to striate cortex damage) despite the absence of perceptual awareness of the faces. Functional MRI in this patient revealed that amygdala activation for fearful and nonfearful faces presented in the blind hemifield was comparable with that found in normal sighted subjects (Morris et al., 1996; Morris et al., 2001). Most interestingly with respect to patient $\mathrm{MB}$, it was shown that two subcortical structures - the superior colliculus and the posterior thalamus - are coactivated with the amygdala during the presentation of fearful stimuli in the patient with affective blindsight (Morris et al., 2001; see also Morris, Öhman, \& Dolan, 1999 for results in healthy subjects). Both latter structures are heavily connected with the dorsal pathway (Robinson \& McClurkin, 1989; Sparks \& Hartwich-Young, 1989) and implicated - together with the dorsal pathway - in the generation of fast, mostly unaware, visuospatial and visuomotor behaviour (Grüsser \& Landis, 1991; Milner \& Goodale, 1995). The present case of dog phobia suggests that the parietal lobes are also involved in fear processing and the generation of a specific phobia. We do not know whether dog phobia was caused by direct 
effects of parietal lobe damage on amygdala processing or by more indirect effects via interference with the processing in the medial prefrontal cortex, the thalamus, or the superior colliculus (see Figure 1B). Whereas the two latter subcortical structures have been shown to be coactivated by fearful stimuli, the medial prefrontal cortex has been proposed to be involved in fear extinction and modulation of amygdala responsiveness (LeDoux, 1996; see also Bremner et al., 1999). In conclusion, the present observation suggests that irrational fear, as found in specific phobias, might not only result from a dissociation of aware cortical from unaware subcortical fear responses, but also from a selective uncoupling of unaware cortical fear responses from aware cortical and unaware subcortical fear responses.

Revised manuscript received 25 October 2002

\section{REFERENCES}

Adolphs, R., Tranel, D., Damasio, H., \& Damasio, A. (1994). Impaired recognition of emotion in facial expressions following bilateral damage to the human amygdala. Nature, 3472, 669-372.

Aggleton, J. P. (1992). The functional effects of amygdala lesions in humans: A comparison with findings from monkeys. In J.P. Aggleton (Ed.), The amygdala: Neurobiological aspects of emotion, and mental dysfunction (pp. 485-503). New York: Wiley-Liss.

Agras, W. S., Chapin, H. N., \& Oliveau, D. C. (1972). The natural history of phobia. Course and prognosis. Archives of General Psychiatry, 26, 315-317.

Amaral, D. G., Price, D. L., Pitkanen, A., \& Carmichael, S. T. (1992). Anatomical organization of the primate amygdaloid complex. In J. P. Aggleton (Ed.), The amygdala: Neurobiological aspects of emotion, and mental dysfunction (pp. 1-66). New York: Wiley-Liss.

Birbaumer, N., Grodd, W., Diedrich, O., Klose, U., Erb, M., Lotze, M., Schneider, F., Weiss, U., \& Flor, H. (1998). fMRI reveals amygdala activation to human faces in social phobics. NeuroReport, 9, 1223-1226.

Blanke, O., Landis, T., Safran, A. B., \& Seeck, M. (2002). Direction specific motion blindness induced by focal stimulation of human extrastriate cortex. European Journal Neuroscience, 15, 2043-2048.

Bremner, J. D., Staib, L. H., Kaloupek, D., Southwick, S. M., Soufer, R., \& Charney, D. S. (1999). Neural correlates of exposure to traumatic pictures and sound in Vietnam combat veterans with and without posttraumatic stress disorder: A positron emission tomography study. Biological Psychiatry, 45, 806-816.

Calder, A. J., Young, A. W., Rowland, D., Perret, D. I., Hodges, J. R., \& Etcoff, N. L. (1996). Facial emotion recognition after bilateral amygdala damage: Differentially severe impairment of fear. Cognitive Neuropsychology, 13, 699-745.

Fyer, A. J. (1998). Current approaches to etiology and pathophysiology of specific phobias. Biological Psychiatry, 44, 1295-1304.

Goldstein, K., \& Gelb, A. (1918). Psychologische Analysen hirnpathologischer Fälle auf Grund von Untersuchungen Hirnverletzter: I. Abhandlung. Zur Psychologie des optischen Wahrnehmungsund Erkenungsvorganges. Zeitschrift für die gesamte Neurologie und Psychiatrie, 41, 1-142.

Grüsser, O. J., \& Landis, T. (1991). Visual movement agnosia or motion blindness: A rare clinical symptom. In J. Cronly-Dillon (Ed.), Visual agnosias and other disturbances of visual perception and cognition.Vision and visual dysfunction (Vol. 12, pp. 359-384). London: Macmillan. 
LeDoux, J. (1996). The emotional brain: The mysterious underpinnings of the emotional life (pp. 141-165, 252-256). New York: Simon \& Schuster.

Liberzon, I., Taylor, S. F., Amdur, R., Jung, T. D., Chamberlain, K. R., Minoshima, S., Koeppe, R. A., \& Fig, L. M. (1999). Brain activation in PTSD in response to trauma-related stimuli. Biological Psychiatry, 45, 817-826.

Losey, F., Safran, A. B., Mermoud, C., Michel, C. M., \& Landis, T. (1998). Perception visuelle du mouvement. Etude normative. Klinische Monatsblätter für Augenheilkunde, 212, 379-381.

Milner, A. D., \& Goodale, M. A. (1995). The visual brain in action. Amsterdam: Elsevier.

Morris, J. S., Frith, C. D., Perret, D. I., Rowland, D., Young, A. W., Calder, A. J., \& Dolan, R. J. (1996). A differential neural response in the human amygdala to fearful and happy facial expressions. Nature, 383, 812-815.

Morris, J. S., DeGelder, B., Weiskrantz, L., \& Dolan, R.J. (2001). Differential extrageniculostriate and amygdala responses to presentation of emotional faces in a cortically blind field. Brain, 124, 1241-1252.

Morris, J. S., Öhman, A., \& Dolan, R. J. (1999). A subcortical pathway to the right amygdala mediating "unseen", fear. Proceedings of the National Academy Science, USA, 96, 1680-1685.

Rauch, S. L., Whalen, P. J., Shin, L. M., McInerney, S. C., Macklin, M. L., Lasko, N. B., Orr, S. P., \& Pitman, R. K. (2000). Exaggerated amygdala response to masked facial stimuli in posttraumatic stress disorder: A functional MRI study. Biological Psychiatry, 47, 769-776.

Robinson, D. L., \& McClurkin, J. W. (1989). The visual superior colliculus and pulvinar. In R.H. Wurtz \& M.E. Goldberg (Eds.), The neurobiology of saccadic eye movements (pp. 337-360). New York: Elsevier.

Seligman, M. E. (1971). Phobias and preparedness. Behavior Therapy, 2, 307-320.

Shin, L. M., Kosslyn, S. M., McNally, R. J., Alpert, N. M., Thompson, W. L., Rauch, S. L., Macklin, M. L., \& Pitman, R. K. (1997). Visual imagery and perception in posttraumatic stress disorder: A positron emission tomographic investigation. Archives of General Psychiatry, 54, 233-241.

Sparks, D. L., \& Hartwich-Young, R. (1989). The deep layers of the superior colliculus. In R. H. Wurtz \& M. E. Goldberg (Eds.), The neurobiology of saccadic eye movements (pp. 213-255). New York: Elsevier.

Ungerleider, M. G., \& Miskin, M. (1982). Two cortical visual systems. In D. J. Ingle, M. A. Goodale, R. J. Mansfield (Eds.), Analysis of visual behavior (pp. 549-580). Cambridge, MA: MIT Press.

Zihl, J., von Cramon, D., \& Mai, N. (1983). Selective disturbance of movement vision after bilateral brain damage. Brain, 106, 313-340. 
Copyright $\odot 2003$ EBSCO Publishing 